\title{
Expression regulation and function of NLRC5
}

\author{
Yikun Yao, Youcun Qian ${ }^{凶}$ \\ The Key Laboratory of Stem Cell Biology, Institute of Health Sciences, Shanghai Institutes for Biological Sciences, Chinese \\ Academy of Sciences/Shanghai Jiao Tong University School of Medicine, Shanghai 200025, China \\ $\triangle$ Correspondence: ycqian@sibs.ac.cn \\ Received October 30, 2012 Accepted December 10, 2012
}

\begin{abstract}
The NOD like receptors (NLRs), a class of intracellular receptors that respond to pathogen attack or cellular stress, have gained increasing attention. NLRC5, the largest member of the NLR protein family, has recently been identified as a critical regulator of immune responses. While NLRC5 is constitutively and widely expressed, it can be dramatically induced by interferons during pathogen infections. Both in vitro and in vivo studies have demonstrated that NLRC5 is a specific and master regulator of major mistocompatibility complex (MHC) class I genes as well as related genes involved in MHC class I antigen presentation. The expression of MHC class I genes is regulated by NLRC5 in coordination with the RFX components through an enhanceosome-dependent manner. And the involvement of NLRC5 in MHC class I mediated CD8 ${ }^{+}$ $\mathrm{T}$ cell activation, proliferation and cytotoxicity is proved to be critical for host defense against intracellular bacterial infections. Nevertheless, the role of NLRC5 in innate immunity remains to be further explored. Here, we review the research advances on the structure, expression regulation and function of NLRC5.
\end{abstract}

\section{KEYWORDS NLR, NLRC5, MHC Class I}

\section{INTRODUCTION}

Harmful exogenous pathogens removal is extremely critical for human beings as living in an environment full of microorganisms. In order to efficiently recognize and resist the attack from harmful microbes, our body has evolved two systems, innate and adaptive immune systems. The innate immune system is non-specific and the first line of defense against foreign organism invasion. Pattern Recognition Receptors (PRRs) are critical sensors in the innate immune system to detect exogenous microbial invasion and cellular stress. NOD Like Receptors (NLRs) are a recently discovered family of cytoplasmic PRRs whose function is mainly to induce inflammation and cell death, facilitating quick removal of invasive pathogens. Meanwhile, after these foreign pathogens are endocytosed by innate immune cells, fragments of microbial proteins are processed and presented to $\mathrm{T}$ lymphocytes by major histocompatibility complex (MHC) class I or MHC class II. Those T lymphocytes bearing antigen-specific $\mathrm{T}$ cell receptors (TCRs) then expand through the interaction with MHC class I or II. Antigen presentation by $\mathrm{MHC}$ class II is pivotal for the activation of $\mathrm{CD}^{+} \mathrm{T}$ cells as MHC class I is for $\mathrm{CD} 8^{+} \mathrm{T}$ cells. Both antigen-specific $\mathrm{CD}^{+}$and $\mathrm{CD}^{+} \mathrm{T}$ cells play key roles in clearing pathogens efficiently and specifically.

Over a decade ago, it was reported that the class II transactivator (CIITA also known as NLRA), was essential for conventional $\mathrm{MHC}$ class II gene expression and consequently $\mathrm{CD}^{+} \mathrm{T}$ cell development and activation through genetic study of Ciita-deficient mice (Chang et al., 1996). Therefore, CIITA represents a master regulator of MHC class II gene expression (Williams et al., 1998; LeibundGut- Landmann et al., 2004). It has recently been discovered that another NLR family member NLRC5 (NLR family, CARD domain containing 5, also called NOD4 or NOD27), is important for the expression of MHC class I genes and associated immune functions.

NLRC5 is a newly identified member of the NLR family which contains more than 20 members in mammalian genome, and even more in plant or some fish species (Martinon et al., 2009; Schroder and Tschopp, 2010). These family members are generally divided into three subfamilies: the NLRCs (with CARD domain in its N-terminal), NAIPs (with BIR domain in its N-terminal) and the NLRPs (with PYD domain in its $\mathrm{N}$ terminal). NLRC subfamily contains five members NLRC1NLRC5 while NLRPs consist of at least 14 members NLRP1NLRP14. Among the NLRC subfamily, NLRC1 (also known as NOD1), NLRC2 (also NOD2) and NLRC4 are well studied and are shown to play critical roles in host defense against bacterial pathogens (Strober et al., 2006; Gong and Shao, 2012). NLRP3 is the most extensively investigated member of the NLRP subfamily. NLRP3 sense both pathogen associ- 
ated molecular patterns (PAMPs) and danger signals to form inflammasome for IL-1b maturation (Tschopp and Schroder, 2010). Recent studies have shed light on the role of other NLR members. NLRP1 senses Bacillus anthracis lethal toxinforms to form inflammasome (Levinsohn et al., 2012). NLRP6 actives inflammasome for IL-18 maturation to alleviate colitis but negatively regulates Toll-Like Receptor (TLR) signaling pathways against bacterial pathogens (Elinav et al., 2011; Anand et al., 2012). NLRP12 also plays dual roles in immunity. It functions asYersinia pestis recognition and inflammasome formation for IL-1b and IL-18 maturation for host defense. Meanwhile, it also suppresses colon inflammation and tumorigenesis through negatively regulating the canonical or non-canonical NF-kB signaling (Zaki et al., 2011; Allen et al., 2012; Vladimer et al., 2012). NLRP7 forms inflammasome during recognition of microbial lipopeptides in human macrophages (Khare et al., 2012). NLRP4 plays a negative role in the regulation of type I interferon signaling and autophagy (Jounai et al., 2011; Cui et al., 2012). NLRP10 is essential to initiate adaptive immunity by influencing the intrinsic function of dendritic cells (Eisenbarth et al., 2012). NLRC3 inhibits TLR4 mediated NF-kB activation and inflammation (Schneider et al., 2012). NLRC5 is a critical regulator of MHC Class I genes for host defense against bacterial infection (Biswas et al., 2012; Yao et al., 2012). Generally, NLRs play various roles in regulating immunity and inflammation.

This review focuses on the recent studies of NLRC5 and highlights its function in regulation of MHC class I genes.

\section{STRUCTURE OF NLRC5}

Human NLRC5 is located in the $16 q 13$ locus and consists of 1866 amino acids (aa) while mouse NLRC5 is at chromosome 8 and contains 1915 aa. There is an identity up to $64 \%$ between human and mouse NLRC5. Similar to other NLRs, NLRC5 contains three structural domains including the Nterminal atypical caspase activation and recruitment domain (CARD), the centrally located NACHT (named after NAIP, CIITA, HET-E, and TP-1 proteins) and 27 leucine-rich repeats (LRRs) at the C-terminal by structure prediction analyses (Benko et al., 2010). The exon 1-3 together and exon 4 of NLRC5 gene encode the CARD domain and the NACHT domain respectively while the rest exons encode the LRR domain. Due to its unusually long stretch of C-terminal LRRs, NLRC5 is the largest member in the NLR family with a predicted size of more than $200 \mathrm{kDa}$ while other NLRs typically possess a molecular weight of $80-120 \mathrm{kDa}$. The CARD domain of NLRC5 appears atypical as it shows little similarity with the defined CARD domain found in other NLRCs (Fig. 1). The alignment of the NACHT and LRR domains with other NLR family members reveals that NLRC5 is closely related to CIITA (Benko et al., 2010; Meissner et al., 2010). CIITA has several Nuclear Localization Signals (NLSs) in its N and C terminal region and is involved in a nucleo-cytoplasmic transport behavior that is crucial for its regulation of MHC-II gene transactivation (Cama-
cho-Carvajal et al., 2004). Likewise, NLRC5 also has bipartite NLS in its N terminus and overexpressed NLRC5 was found to be dominantly localized in the nucleus, suggesting a role of NLRC5 in the nuclear (Meissner et al., 2012a). As a matter of fact, endogenous NLRC5 protein was found to be in both cytoplasmic and nuclear while it was mainly localized in the nucleus after treatment with LepB, an inhibitor of protein nucleus export, suggesting NLRC5 is a cytoplasm-nuclear shuttle protein (Neerincx et al., 2012; Staehli et al., 2012). The results of our structural analyses obtained from Ensembl database have predicted 10 protein coding isoforms of NLRC5 (data not shown). Actually, the mRNA levels of some of these isoforms have already been detected and they appears to be differentially expressed in various tissues and cell lines (Neerincx et al., 2012). However, it remains to be determined whether these isoforms really code stable proteins or have any function.

\section{EXPRESSION OF NLRC5}

Given that the molecule expression profiling is helpful in investigating its function, most studies have examined NLRC5 expression pattern in a variety of cells and tissues. Immune tissues/organs like spleen, lymph node, bone marrow and thymus all have high-level expression of NLRC5. NLRC5 is also expressed abundantly in lung and intestine, which have close contact with microorganism. More specifically, NLRC5 is found highly expressed in immune cells including mouse primary cells such as bone marrow-derived macrophages (BMDMs), splenic DCs, $C D 4^{+} \mathrm{T}$ cells, $\mathrm{CD} 8^{+} \mathrm{T}$ cells and $\mathrm{B}$ cells as well as in cell lines such as human THP-1, Jurkat T cells, Raji B cells and mouse RAW264.7 (Benko et al., 2010; Cui et al., 2010; Neerincx et al., 2010). While NLRC5 is constitutively expressed in many immune cells, it can also be dramatically induced by a variety of stimulations. Interferon- $\mathrm{Y}$ (IFN- $\mathrm{\gamma}$ ) is a widely reported stimulus capable of strongly inducing NLRC5 expression at both mRNA and protein level in various cell types like lymphocytes $\left(\mathrm{CD} 4^{+} \mathrm{T}\right.$ cells, $\mathrm{CD} 8^{+} \mathrm{T}$ cells), myeloid cells (macrophage or dendritic cells), fibroblasts and Hela cells (Benko et al., 2010; Neerincx et al., 2010; Staehli et al., 2012). LPS is also confirmed to induce a high level of NLRC5 transcription and expression (Cui et al., 2010; Staehli et al., 2012; Yao et al., 2012). Interestingly, this up-regulation was dependent on TRIF but not MyD88, which were adaptors of TLR4 downstream signaling and mediate type I interferon (IFN) producing pathway and NF-KB activation pathway respectively, implying that type I interferon may induce NLRC5 expression. Indeed, this hypothesis is demonstrated in innate immune cells like macrophages (Staehli et al., 2012). Consistent with that, the induction of NLRC5 expression by LPS was abrogated in Ifnar ${ }^{-1-}$ bone marrow-derived macrophages (Staehli et al., 2012). NLRC5 promoter region is predicted to have STAT1 and NF-KB binding sites (Kuenzel et al., 2010). Accordingly, NLRC5 induction is reported to be severely reduced in Stat ${ }^{-1-}$ BMDMs after treatment of interferons or various stimuli which can induce interferons such as LPS. Likewise, the expression 


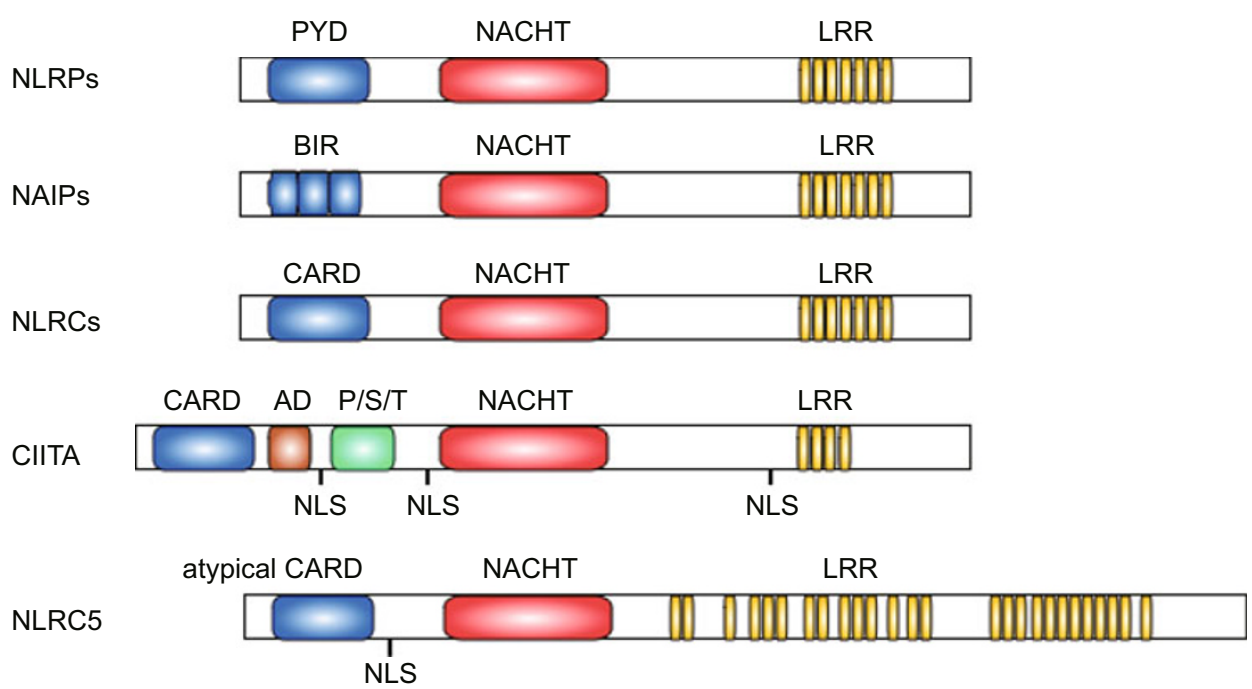

Figure 1. The structural domains of NLRC5. The typical domains of the NLR subfamilies (NLRPs, NAIPs, NLRCs and CIITA) are shown for comparison with NLRC5. NLRC5 has typical tripartite domains including the N-terminal atypical caspase activation and recruitment domain (CARD), the centrally located NACHT (named after NAIP, CIITA, HET-E, and TP-1 proteins) and multiple leucinerich repeats (LRRs) at the C-terminal. The NLR subfamilies are classified by the differential N-terminal domains: the NLRCs with CARD domain, NAIPs with BIR domain and the NLRPs with PYD domain. NACHT (also known as NBD domain): named after NAIP, CIITA, HET-E, and TP-1 proteins. LRR: leucine-rich repeats. AD: acidic domain or activation domain. P/S/T domain: proline-serine-threonine-rich domain. NLS: nuclear localization signal. CARD: caspase recruitment domain. PYD: pyrin domain.

of NLRC5 was impaired in Stat ${ }^{-1-}$ T cells after TCR activation which can promote IFN-y production. Based on these discoveries, STAT1 is suggested to play an essential role in the regulation of NLRC5 expression (Staehli et al., 2012). NLRC5 has also been observed to be induced by diversified bacteria and virus, or their PAMPs like poly(I:C), CpG and poly(dA:dT) which all can induce type I IFN (Cui et al., 2010; Kuenzel et al., 2010; Staehli et al., 2012; Yao et al., 2012). Therefore, both type I and type II IFN can strongly induce NLRC5 expression through STAT1 dependent pathway.

\section{ROLES OF NLRC5 IN REGULATING IMMUNE RESPONSES}

\section{NLRC5 is a master regulator of MHC class I gene expression}

MHC class I molecules, a family of cell surface receptors, mainly bind endogenous antigen peptides in almost all nucleated cells, and are comprised of a polymorphic membrane-linked heavy chain and a shared soluble light chain ( $\beta 2$-microglobulin; $\beta 2 \mathrm{~m})$. MHC class I heavy chains contain the more polymorphic classical (class la) HLA-A, HLA-B, and HLA-C leukocyte antigens in humans and $\mathrm{H}-2 \mathrm{~K}, \mathrm{H}-2 \mathrm{D}$, and $\mathrm{H}-2 \mathrm{~L}$ in mice, and the less polymorphic nonclassical (class Ib) HLA-E, HLA-F and HLA-G antigens in humans and H2-Qa1, Tla and H2-M3 in mice. In humans, the genes encoding the MHC class I heavy chains are located within a MHC super-locus on chromosome 6 , a region associated with more than 100 different diseases, including diabetes, asthma and various autoimmune disorders like rheumatoid arthritis and psoriasis (Lie and Thorsby, 2005), while the gene encoding $\beta 2 \mathrm{~m}$ is located on chromosome 15 . In mice, the genes for heavy chains and $\beta 2 \mathrm{~m}$ are located in chromosome 17 and chromosome 2 respectively.

MHC class I antigen processing and presentation can be separated into three major steps. Firstly, protein antigens, such as self-proteins, tumor antigens and intracellular pathogens in the cytosol, are proteolytically lized into antigenic peptides through the proteasome containing low molecular mass protein 2 (LMP2) and low molecular mass protein 7 (LMP7), another two genes that located within the MHC locus. Secondly, the antigenic peptides are translocated from cytosol to the endoplasmic reticulum (ER) by the transporter associated with antigen processing (TAP), a gene located in the MHC locus as well (Shepherd et al., 1993). The assembly of MHC class I molecules and peptides then occurs in the ER. Thirdly, the MHC class I-peptide complexes migrate through the Golgi body to the surface of antigen presenting cells to mediate MHC class I restricted activation of effector cells such as CD8 ${ }^{+}$ T cells (Pamer and Cresswell, 1998; Peaper and Cresswell, 2008).

As the largest member of NOD-like receptor family, NLRC5 has been widely found to be involved in control of MHC class I gene expression in recent intensive studies (Kobayashi and van den Elsen, 2012; Zhao and Shao, 2012). Initial studies found that NLR family member CIITA could regulate the MHC class I gene activation through overexpression system in vitro (Martin et al., 1997; Williams et al., 2003). However, the expression of MHC class I genes was not affected in Ciita-deficient mice (Chang et al., 1996), suggesting that there may be unidentified regulators for $\mathrm{MHC}$ class I genes. As mentioned 
above, sequence analysis indicates that NLRC5 is most similar to CIITA, a master regulator of MHC class II gene expression, which interacts with transcription factors to form a complex called MHC enhanceosome (Masternak and Reith, 2002). What is more, with the requirement of their NLS, they can both localize to nucleus where NLRC5 is possibly engaged in gene expression and regulation. Indeed, it was found that overexpression of wild-type NLRC5 instead of its NLS mutated forms in Jurkat $\mathrm{T}$ cell lines could induce expression of various $\mathrm{MHC}$ class I family genes as well as genes related with class I antigen processing and presentation, including HLA-A,B,C,E,F,G; ß2M, TAP1, LMP2 (Meissner et al., 2010). Besides, overexpression of NLRC5 did not induce expression of MHC class II genes, revealing its specificity in gene regulation (Meissner et al., 2010). Further overexpression assay showed that all the structural domains of NLRC5 including CARD, NACHT and LRR, were required for MHC class I gene induction (Meissner et al., 2010, 2012a). The results of the overexpression assays were confirmed by siRNA mediated NLRC5 knock-down studies (Meissner et al., 2010).

How does NLRC5 regulate MHC class I gene expression? It has already been shown CIITA regulates $\mathrm{MHC}$ class II gene expression through directly affecting the gene promoters (Meissner et al., 2010; Staehli et al., 2012). Both MHC class I and MHC class II genes have a conserved WXY motif in their promoter regions (Moreno et al., 1999). As expected, ChIP assay indicated that NLRC5 associated with the MHC class I promoter in a similar manner as CIITA with MHC class II promoter (Meissner et al., 2010). It was well studied that the MHC class II gene transcription requires formation of the MHC class II enhanceosome containing the transcription regulators RFX complex in $\mathrm{MHC}$ class II promoters. Other factors belonging to the MHC class II enhanceosome like CREB and NF-Y assemble on the MHC-II promoter and bind to the conserved $\mathrm{X} 1, \mathrm{X} 2$, and $Y$ boxes respectively (Hake et al., 2000; Jabrane-Ferrat et al., 2003). Similarly, NLRC5-mediated MHC class I promoter activation also requires the W/S and $\mathrm{X} 1, \mathrm{X} 2$ cis-regulatory elements and the involvement of MHC class I enhanceosome (Neerincx et al., 2012). Further analyses showed that NLRC5 interacts with the transcription regulator RFXANK/B which is further associated with the transcription factors RFX5 and RFXAP to form the RFX complex involved in the regulation of MHC class I promoter (Meissner et al., 2012b). It is also reported that NLRC5 could affect histone methylation (H3K27me3), suggesting NLRC5 may have the ability to influence gene expression through adjusting the chromosome activation status at the MHC class I locus (Meissner et al., 2012b). The nuclearcytoplasmic shuttling process of NLRC5 is also reported to be important for $\mathrm{MHC}$ class I gene promoter activation in an overexpression system (Meissner et al., 2012a). Despite of these pioneer studies, how NLRC5 regulates its target gene promoters exactly remains to be determined. As mentioned above, $\beta 2 \mathrm{M}$ is not in the $\mathrm{MHC}$ class I gene locus but is regulated by NLRC5. It will be interesting to know how NLRC5 exactly regulates $\beta 2 \mathrm{M}$ gene expression. As the known mechanism of
NLRC5 in regulating MHC class I gene expression is similar to that of CIITA in regulation of MHC class II genes, it will also be interesting to understand how NLRC5 and CIITA exert their influence on gene expression specifically. MHC class I-peptide bearing cells can interact with CD8 cytotoxic T lymphocytes (CTL) through the corresponding $\mathrm{MHC}$ molecule and the T-cell receptor (TCR) to mediate $\mathrm{CD}^{+} \mathrm{T}$ cell activation and cytokine production, proliferation, and lysis of target cells (Savage et al., 1999; Jiang and Chess, 2000). The key finding that NLRC5 regulates $\mathrm{MHC}$ class I gene expression in vitro was confirmed by in vivo mouse genetic studies (Biswas et al., 2012; Robbins et al., 2012; Staehli et al., 2012; Tong et al., 2012; Yao et al., 2012). By generating NIrc5-deficient mice independently, we and other groups recently reported that the expression of $\mathrm{MHC}$ class I genes $(\mathrm{H}-2 \mathrm{D}, \mathrm{H}-2 \mathrm{~K}, \mathrm{H}-2 \mathrm{M} 3, \mathrm{H}-2 \mathrm{Qa}, \beta 2 \mathrm{M})$ and $\mathrm{MHC}$ class I related genes (LMP2, TAP1) were reduced in NIrc5deficient spleen and thymus compared with wild-type control mice (Biswas et al., 2012; Robbins et al., 2012; Staehli et al., 2012; Tong et al., 2012; Yao et al., 2012). In subsequent cell type analyses, the constitutive expression of both the classical and nonclassical MHC class I genes was dramatically impaired in NLRC5 deficient T, NKT, NK and $\mathrm{\delta}$ T lymphocytes, and moderately reduced in NLRC5 deficient B cells, conventional dendritic cells (cDCs), cortical and medullary thymic epithelial cells (TECs) (Biswas et al., 2012; Robbins et al., 2012; Staehli et al., 2012). All these data suggest that NLRC5 regulates the expression of MHC class I genes in different cell types. In contrast, the MHC class II gene expression was not affected in Nlrc5-deficient mice (Robbins et al., 2012), confirming that NLRC5 specifically regulates MHC class I genes. As discussed above, NLRC5 is induced by PAMPs (LPS, poly(I:C) and poly(dA:dT)) and IFN- $\gamma$ which all can increase MHC class I gene expression. We and others found that these PAMPs and IFN-y induced expression of MHC class I genes was also reduced upon NLRC5 deficiency (Biswas et al., 2012; Robbins et al., 2012; Staehli et al., 2012; Yao et al., 2012). Thus, NLRC5 specifically regulates both constitutive and inducible expression of $\mathrm{MHC}$ class I genes.

MHC class I complex is essential for MHC class I antigen presentation to $\mathrm{CD}^{+} \mathrm{T}$ cells for their activation, proliferation and cytotoxicty. Consistent with the fact that NLRC5 mediated regulation of $\mathrm{MHC}$ class I genes, we recently found that $\mathrm{MHC}$ class I antigen specific $\mathrm{CD} 8^{+} \mathrm{T}$ cell activation, proliferation and lysis of target cells were impaired upon NLRC5 deficiency (Yao et al., 2012). MHC class I mediated antigen presentation and $\mathrm{CD}^{+} \mathrm{T}$ cell activation is critical for host defense against intracellular bacterial and viral pathogens. We and Kobayashi group both found that NLRC5 was crucial for host defense against intracellular bacteria L. monocytogenes infection (Biswas et al., 2012; Yao et al., 2012). The induction of MHC class I gene expression was observed to be impaired in BMDMs in vitro and in mice in vivo after infection with L. monocytogenes (Yao et al., 2012). Consistently, activated $C D 8^{+} \mathrm{T}$ cell numbers and IFN-y production were dramatically reduced in NLRC5 deficient mice after infection although uninfected Nlrc5-deficient 
mice showed minor differences in $\mathrm{CD} 8^{+} \mathrm{T}$ cell percentages in the periphery including spleen, liver, lymph nodes, and blood (Staehli et al., 2012; Yao et al., 2012). Moreover, NLRC5deficient mice also failed to activate $L$. monocytogenes-specific $\mathrm{CD}^{+} \mathrm{T}$ cell responses, accompanied with less IFN-y secretion and increased bacterial loads in spleen and liver (Biswas et al., 2012; Yao et al., 2012). Accordingly, the bacterial load was significantly increased in spleen and liver of NLRC5 deficient mice after infection with $\mathrm{L}$. monocytogenes. MHC class I mediated $\mathrm{CD}^{+} \mathrm{T}$ cell activation also contributes to anti-cancer immunity (Vesely et al., 2011). The reduction of tumor cell surface MHC class I gene expression results in immune surveillance evasion. The level of NLRC5 expression was actually shown to be low in several T and B cell derived tumor cell lines (Staehli et al., 2012), suggesting tumor cells may evade immune surveillance through NLRC5 downregulation. While NLRC5 is well defined to mediate MHC class I restricted anti-bacterial immunity, its roles in MHC class I associated anti-viral immunity, anti-tumor immunity and graft transplantation still remain to be further explored.

\section{Function of NLRC5 in innate immune response}

While the role of NLRC5 in regulating MHC class I gene expression is well recognized by intensive studies, previous researches suggested that NLRC5 may also regulate innate immune responses through affecting TLR mediated NF-KB activation, type I interferon (IFN)-producing pathways as well as inflammasome activation (Benko et al., 2010; Cui et al., 2010; Neerincx et al., 2010; Davis et al., 2011; Lamkanfi and Kanneganti, 2012).Two studies showed NLRC5 was a positive regulator of type I IFN producing pathway in human fibroblasts and THP-1 cells (Kuenzel et al., 2010; Neerincx et al., 2010). However, NLRC5 was shown to suppress type I IFN production through association with RIG-I and MDA5 (the dsRNA sensors), or inhibit NF-KB pathway through its direct binding with IKB kinase- $\alpha$ (IKK- $\alpha$ ) and IKK- $\beta$ (Cui et al., 2010). The cause of the discrepancy is probably the differential study conditions. The negative regulation of NLRC5 on type I IFN production and NF-KB activation in the in vitro system is supported by one in vivo study of NLRC5 deficient mice in which exon 8 of NLRC5 gene is deleted (Tong et al., 2012). The negative roles of NLRC5 appear to be cell type specific because IL-6 and IFN- $\beta$ level were detected higher in NIrc5-deficient mouse embryonic fibroblasts (MEFs), peritoneal macrophages and bone marrow-derived macrophages, but not in NLRC5 deficient BMDCs after TLR stimulation or VSV infection (Tong et al., 2012). However, the inhibitory roles of NLRC5 in type I IFN production and NF-KB were not observed in the other NLRC5 deficient mice in which exon 4, exon 3-4 or exon 1-4 was selected to be targeted by several groups (Kumar et al., 2010; Robbins et al., 2012; Staehli et al., 2012; Yao et al., 2012). The discrepancy is likely due to the differential targeting strategies. We and Ting's group reported NLRC5 interacted with NLRP3 and promoted the inflammasome activation in human cell lines in vitro
(Davis et al., 2011; Yao et al., 2012). We observed that NLRP3 mediated IL-1b production was partially impaired upon NLRC5 deficiency, but Ting's group did not find this phenotype. The discrepancy is also likely due to differential targeting strategies as deletion of different exons of NLRC5 may lead to potential truncated and differently spliced isoforms. To date, the role of NLRC5 in regulating innate immune responses is unclear and remains to be further explored.

\section{FUTURE PERSPECTIVES}

Recent studies have identified NLRC5 as a critical immune regulator. NLRC5 specifically regulates transcription of MHC class I genes and associated genes involved in MHC class I antigen presentation, and consequently $\mathrm{CD} 8^{+} \mathrm{T}$ cell activation for host defense against intracellular bacterial infections (Fig. 2). Furthermore, NLRC5 mediated regulation of MHC class I expression has been found through the same MHC enhanceosome components utilized by CIITA in regulating MHC class II gene expression. However, it still remains to be explored how NLRC5 and CIITA specifically regulate MHC class I and II gene expression respectively and it is important to identify potential specific factors mediating their gene regulation. Although the role of NLRC5 in regulating MHC class I gene expression has been well defined, its function in regulating innate immunity is still not clear and that needs to be further investigated. As NLRC5 specifically regulates MHC class I genes, it could be a potential target for transplantation rejection, cancer immunotherapy and vaccine development.

\section{ABBREVIATIONS}

CDC, conventional dendritic cell; CIITA, class II transactivator; ER, endoplasmic reticulum; IFN, interferon; IKK, IKB kinase; LMP, low molecular mass protein; LRR, leucine-rich repeat; MEF, mouse embryonic fibroblast; MHC, major histocompatibility complex; NLR, NOD-like receptor; PAMP, pathogen associated molecular pattern; PRR, Pattern recognition receptor; TAP, transporter associated with antigen processing; TCR, T cell receptor; TEC, thymic epithelial cell; TLR, Toll-like receptor

\section{ACKNOWLEDGEMENTS}

The authors have no conflicting financial interests. Supported by grants from the National Natural Science Foundation of China (Grant Nos. 81230075, 30930084 and 91029708), the National Basic Research Program (973 Program) (Nos. 2013CB944904 and 2010CB529705), and Science and Technology Commission of Shanghai Municipality (No. 10JC1416600)

\section{REFERENCES}

Allen, I.C., Wilson, J.E., Schneider, M., Lich, J.D., Roberts, R.A., Arthur, J.C., Woodford, R.M., Davis, B.K., Uronis, J.M., Herfarth, H.H., et al. (2012). NLRP12 suppresses colon inflammation and tumorigenesis through the negative regulation of noncanonical NF-kappaB signaling. Immunity $36,742-754$. 


\section{Bacteria, virus}

PAMPs such as TLRs ligands, etc

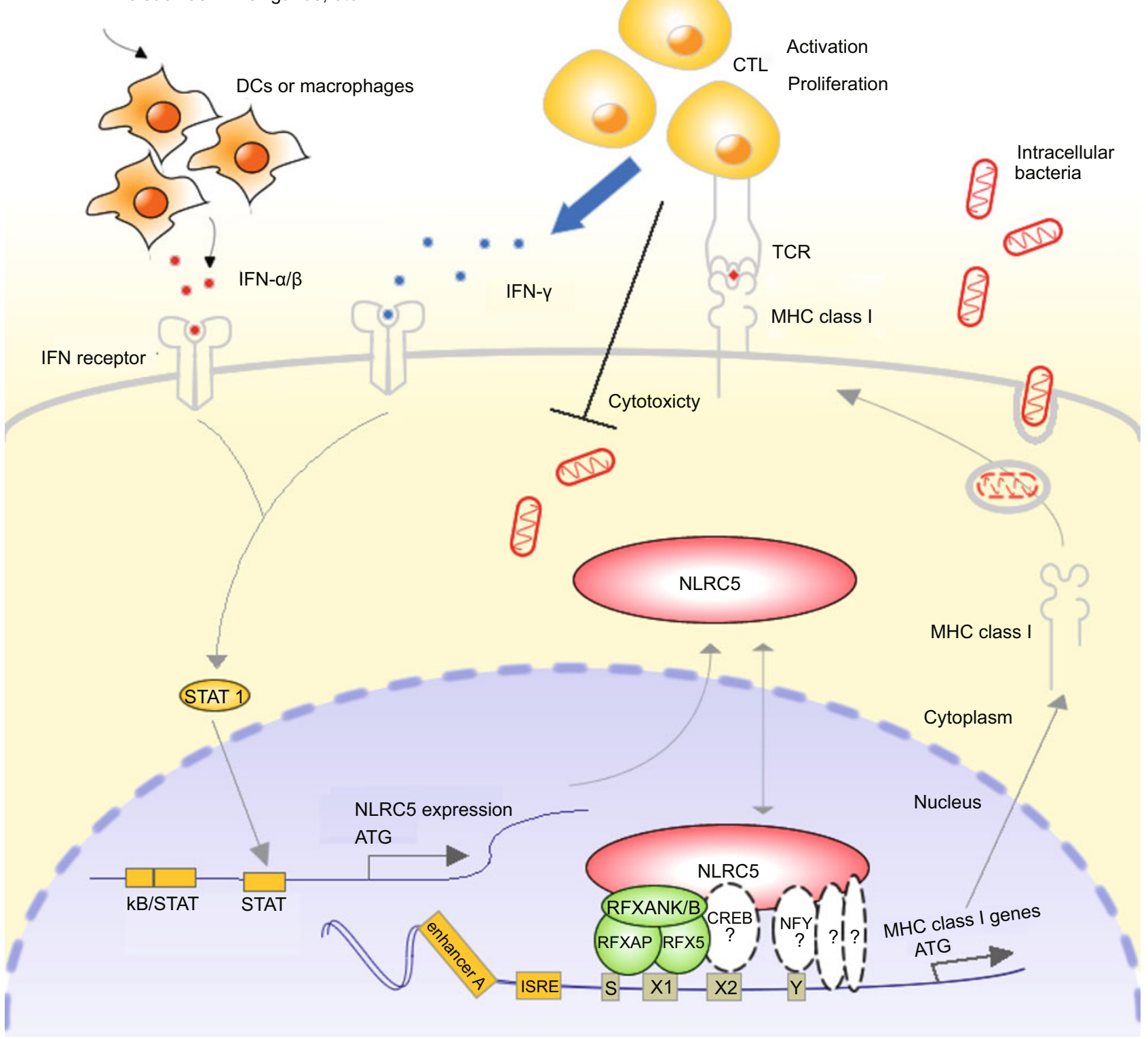

Figure 2. The role of NLRC5 in regulation of MHC class I gene expression in host defense against pathogens. Upon pathogens like intracellular bacteria or virus infection, the PAMPs activate innate immune cells such as dendritic cells (DC) or macrophages to induce type I interferon production (IFN- $\alpha$ and IFN- $\beta$ ). The type I interferons activate the transcription factor STAT1 to up-regulate NLRC5 expression transcriptionally. NLRC5 can shuttle between cytoplasm and nucleus. NLRC5 can form a MHC class I enhanceosome on the MHC class I promoter region through binding RFX component RFXANK/B and other unknown transcription factors to regulate MHC class I gene expression. After intracellular bacterial infections, MHC class I molecules present peptides that are derived from bacterial proteins degraded mainly in the cytosol to the TCR of $C D 8^{+} T$ cells (CTL). This process is essential for MHC class I antigen specific CD8 ${ }^{+} T$ cell activation, proliferation, lysis of target cells to clear the infected bacteria. IFN-y produced by the antigen specific CD8 ${ }^{+} \mathrm{T}$ cells can potently upregulate NLRC5 expression, forming the positive-feedback loop critical for hosts to efficiently eliminate intracellular bacterial pathogens. PAMP: pathogen associated molecular patterns. CTL: cytotoxic T lymphocytes.

Anand, P.K., Malireddi, R.K., Lukens, J.R., Vogel, P., Bertin, J., Lamkanfi, M., and Kanneganti, T.D. (2012). NLRP6 negatively regulates innate immunity and host defence against bacterial pathogens. Nature 488, 389-393.

Benko, S., Magalhaes, J.G., Philpott, D.J., and Girardin, S.E. (2010).
NLRC5 limits the activation of inflammatory pathways. J Immunol 185, 1681-1691.

Biswas, A., Meissner, T.B., Kawai, T., and Kobayashi, K.S. (2012). Cutting edge: impaired $\mathrm{MHC}$ class I expression in mice deficient for nIrc5/class I transactivator. J Immunol 189, 516-520. 
Camacho-Carvajal, M.M., Klingler, S., Schnappauf, F., Hake, S.B., and Steimle, V. (2004). Importance of class II transactivator leucinerich repeats for dominant-negative function and nucleo-cytoplasmic transport. Int Immunol 16, 65-75.

Chang, C.H., Guerder, S., Hong, S.C., van Ewijk, W., and Flavell, R.A. (1996). Mice lacking the MHC class II transactivator (CIITA) show tissue-specific impairment of MHC class II expression. Immunity 4, 167-178.

Cui, J., Li, Y., Zhu, L., Liu, D., Songyang, Z., Wang, H.Y., and Wang, R.F. (2012). NLRP4 negatively regulates type I interferon signaling by targeting the kinase TBK1 for degradation via the ubiquitin ligase DTX4. Nat Immunol 13, 387-395.

Cui, J., Zhu, L., Xia, X., Wang, H.Y., Legras, X., Hong, J., Ji, J., Shen, P., Zheng, S., Chen, Z.J., et al. (2010). NLRC5 negatively regulates the NF-kappaB and type I interferon signaling pathways. Cell 141, 483-496.

Davis, B.K., Roberts, R.A., Huang, M.T., Willingham, S.B., Conti, B.J., Brickey, W.J., Barker, B.R., Kwan, M., Taxman, D.J., Accavitti-Loper, M.A., et al. (2011). Cutting Edge: NLRC5-Dependent Activation of the Inflammasome. J Immunol 186, 1333-1337.

Eisenbarth, S.C., Williams, A., Colegio, O.R., Meng, H., Strowig, T., Rongvaux, A., Henao-Mejia, J., Thaiss, C.A., Joly, S., Gonzalez, D.G., et al. (2012). NLRP10 is a NOD-like receptor essential to initiate adaptive immunity by dendritic cells. Nature 484, 510-513.

Elinav, E., Strowig, T., Kau, A.L., Henao-Mejia, J., Thaiss, C.A., Booth, C.J., Peaper, D.R., Bertin, J., Eisenbarth, S.C., Gordon, J.I., et al. (2011). NLRP6 inflammasome regulates colonic microbial ecology and risk for colitis. Cell 145, 745-757.

Gong, Y.N., and Shao, F. (2012). Sensing bacterial infections by NAIP receptors in NLRC4 inflammasome activation. Protein Cell 3, 98-105.

Hake, S.B., Masternak, K., Kammerbauer, C., Janzen, C., Reith, W., and Steimle, V. (2000). CIITA leucine-rich repeats control nuclear localization, in vivo recruitment to the major histocompatibility complex (MHC) class II enhanceosome, and MHC class II gene transactivation. Mol Cell Biol 20, 7716-7725.

Jabrane-Ferrat, N., Nekrep, N., Tosi, G., Esserman, L., and Peterlin, B.M. (2003). MHC class II enhanceosome: how is the class II transactivator recruited to DNA-bound activators? Int Immunol 15, 467-475

Jiang, H., and Chess, L. (2000). The specific regulation of immune responses by $\mathrm{CD} 8+\mathrm{T}$ cells restricted by the MHC class IB molecule, QA-1. Annu Rev Immunol 18, 185-216.

Jounai, N., Kobiyama, K., Shiina, M., Ogata, K., Ishii, K.J., and Takeshita, F. (2011). NLRP4 negatively regulates autophagic processes through an association with beclin1. J Immunol 186, 1646-1655.

Khare, S., Dorfleutner, A., Bryan, N.B., Yun, C., Radian, A.D., de Almeida, L., Rojanasakul, Y., and Stehlik, C. (2012). An NLRP7containing inflammasome mediates recognition of microbial lipopeptides in human macrophages. Immunity 36, 464-476.

Kobayashi, K.S., and van den Elsen, P.J. (2012). NLRC5: a key regulator of MHC class I-dependent immune responses. Nat Rev Immunol 12, 813-820.

Kuenzel, S., Till, A., Winkler, M., Hasler, R., Lipinski, S., Jung, S., Grotzinger, J., Fickenscher, H., Schreiber, S., and Rosenstiel, P. (2010). The nucleotide-binding oligomerization domain-like receptor NLRC5 is involved in IFN-dependent antiviral immune responses. J
Immunol 184, 1990-2000.

Kumar, H., Pandey, S., Zou, J., Kumagai, Y., Takahashi, K., Akira, S., and Kawai, T. (2011). NLRC5 deficiency does not influence cytokine induction by virus and bacteria infections. J Immunol 186, 994-1000.

Lamkanfi, M., and Kanneganti, T.D. (2012). Regulation of immune pathways by the NOD-like receptor NLRC5. Immunobiology 217, 13-16.

LeibundGut-Landmann, S., Waldburger, J.M., Krawczyk, M., Otten, L.A., Suter, T., Fontana, A., Acha-Orbea, H., and Reith, W. (2004). Mini-review: specificity and expression of CIITA, the master regulator of MHC class II genes. Eur J Immunol 34, 1513-1525.

Levinsohn, J.L., Newman, Z.L., Hellmich, K.A., Fattah, R., Getz, M.A., Liu, S., Sastalla, I., Leppla, S.H., and Moayeri, M. (2012). Anthrax lethal factor cleavage of NIrp1 is required for activation of the inflammasome. PLoS Pathog 8, e1002638.

Lie, B.A., and Thorsby, E. (2005). Several genes in the extended human MHC contribute to predisposition to autoimmune diseases. Curr Opin Immunol 17, 526-531.

Martin, B.K., Chin, K.C., Olsen, J.C., Skinner, C.A., Dey, A., Ozato, K., and Ting, J.P.Y. (1997). Induction of MHC class I expression by the MHC class II transactivator CIITA. Immunity 6, 591-600.

Martinon, F., Mayor, A., and Tschopp, J. (2009). The inflammasomes: guardians of the body. Annu Rev Immunol 27, 229-265.

Masternak, K., and Reith, W. (2002). Promoter-specific functions of CIITA and the MHC class II enhanceosome in transcriptional activation. Embo Journal 21, 1379-1388.

Meissner, T.B., Li, A., Biswas, A., Lee, K.H., Liu, Y.J., Bayir, E., Iliopoulos, D., van den Elsen, P.J., and Kobayashi, K.S. (2010). NLR family member NLRC5 is a transcriptional regulator of MHC class I genes. Proc Natl Acad Sci U S A 107, 13794-13799.

Meissner, T.B., Li, A., Liu, Y.J., Gagnon, E., and Kobayashi, K.S. (2012a). The nucleotide-binding domain of NLRC5 is critical for nuclear import and transactivation activity. Biochem Biophys Res Commun 418, 786-791.

Meissner, T.B., Liu, Y.J., Lee, K.H., Li, A., Biswas, A., van Eggermond, M.C., van den Elsen, P.J., and Kobayashi, K.S. (2012b). NLRC5 cooperates with the RFX transcription factor complex to induce MHC class I gene expression. J Immunol 188, 49514958.

Moreno, C.S., Beresford, G.W., Louis-Plence, P., Morris, A.C., and Boss, J.M. (1999). CREB regulates MHC class II expression in a CIITA-dependent manner. Immunity 10, 143-151.

Neerincx, A., Lautz, K., Menning, M., Kremmer, E., Zigrino, P., Hosel, M., Buning, H., Schwarzenbacher, R., and Kufer, T.A. (2010). A role for the human NLR family member NLRC5 in antiviral responses. J Biol Chem 285, 26223-26232.

Neerincx, A., Rodriguez, G.M., Steimle, V., and Kufer, T.A. (2012). NLRC5 controls basal MHC class I gene expression in an MHC enhanceosome-dependent manner. J Immunol 188, 4940-4950.

Pamer, E., and Cresswell, P. (1998). Mechanisms of MHC class I Restricted antigen processing. Ann Rev Immunol 16, 323-358.

Peaper, D.R., and Cresswell, P. (2008). Regulation of MHC Class I Assembly and Peptide Binding. Annu Rev Cell Dev Bi 24, 343-368.

Robbins, G.R., Truax, A.D., Davis, B.K., Zhang, L., Brickey, W.J., and Ting, J.P. (2012). Regulation of class I major histocompatibility complex (MHC) by nucleotide-binding domain, leucine-rich repeat- 
containing (NLR) proteins. J Biol Chem 287, 24294-24303.

Savage, P.A., Boniface, J.J., and Davis, M.M. (1999). A kinetic basis for $T$ cell receptor repertoire selection during an immune response. Immunity 10, 485-492.

Schneider, M., Zimmermann, A.G., Roberts, R.A., Zhang, L., Swanson, K.V., Wen, H., Davis, B.K., Allen, I.C., Holl, E.K., Ye, Z., et al. (2012). The innate immune sensor NLRC3 attenuates Toll-like receptor signaling via modification of the signaling adaptor TRAF6 and transcription factor NF-kappaB. Nat Immunol 13, 823-831.

Schroder, K., and Tschopp, J. (2010). The inflammasomes. Cell 140, 821-832.

Shepherd, J.C., Schumacher, T.N.M., Ashtonrickardt, P.G., Imaeda, S., Ploegh, H.L., Janeway, C.A., and Tonegawa, S. (1993). Tap1Dependent Peptide Translocation in-Vitro Is Atp-Dependent and Peptide Selective. Cell 74, 577-584.

Staehli, F., Ludigs, K., Heinz, L.X., Seguin-Estevez, Q., Ferrero, I., Braun, M., Schroder, K., Rebsamen, M., Tardivel, A., Mattmann, C., et al. (2012). NLRC5 deficiency selectively impairs MHC class I- dependent lymphocyte killing by cytotoxic T cells. J Immunol 188, 3820-3828.

Strober, W., Murray, P.J., Kitani, A., and Watanabe, T. (2006). Signalling pathways and molecular interactions of NOD1 and NOD2. Nat Rev Immunol 6, 9-20.

Tong, Y., Cui, J., Li, Q., Zou, J., Wang, H.Y., and Wang, R.F. (2012). Enhanced TLR-induced NF-kappaB signaling and type I interferon responses in NLRC5 deficient mice. Cell Res 22, 822-835.

Tschopp, J., and Schroder, K. (2010). NLRP3 inflammasome activa- tion: The convergence of multiple signalling pathways on ROS production? Nat Rev Immunol 10, 210-215.

Vesely, M.D., Kershaw, M.H., Schreiber, R.D., and Smyth, M.J. (2011). Natural innate and adaptive immunity to cancer. Annu Rev Immunol 29, 235-271.

Vladimer, G.I., Weng, D., Paquette, S.W., Vanaja, S.K., Rathinam, V.A., Aune, M.H., Conlon, J.E., Burbage, J.J., Proulx, M.K., Liu, Q., et al. (2012). The NLRP12 Inflammasome Recognizes Yersinia pestis. Immunity 37, 96-107.

Williams, G.S., Malin, M., Vremec, D., Chang, C.H., Boyd, R., Benoist, C., and Mathis, D. (1998). Mice lacking the transcription factor CIITA--a second look. Int Immunol 10, 1957-1967.

Williams, K.L., Taxman, D.J., Linhoff, M.W., Reed, W., and Ting, J.P.Y. (2003). Cutting edge: Monarch-1: A pyrin/nucleotide-binding domain/leucine-rich repeat protein that controls classical and nonclassical MHC class I genes. Journal of Immunology 170 , 5354-5358.

Yao, Y., Wang, Y., Chen, F., Huang, Y., Zhu, S., Leng, Q., Wang, H., Shi, Y., and Qian, Y. (2012). NLRC5 regulates MHC class I antigen presentation in host defense against intracellular pathogens. Cell Res 22, 836-847.

Zaki, M.H., Vogel, P., Malireddi, R.K., Body-Malapel, M., Anand, P.K., Bertin, J., Green, D.R., Lamkanfi, M., and Kanneganti, T.D. (2011). The NOD-like receptor NLRP12 attenuates colon inflammation and tumorigenesis. Cancer Cell 20, 649-660.

Zhao, Y., and Shao, F. (2012). NLRC5: a NOD-like receptor protein with many faces in immune regulation. Cell Res 22, 1099-1101. 